\title{
Head-tail instability and Landau damping in bunches with space charge
}

\author{
V. Kornilov and O. Boine-Frankenheim \\ GSI Helmholtzzentrum fuer Schwerionenforschung GmbH, Planckstraße 1, 64291 Darmstadt, Germany
}

(Received 31 May 2010; published 11 November 2010)

\begin{abstract}
Head-tail modes in bunches with space charge are studied using particle tracking simulations. The eigenfrequencies and eigenfunctions of transverse coherent oscillations in a Gaussian bunch are determined and compared with theories. A model for an airbag distribution in a barrier potential gives good predictions for the head-tail spectrum and for eigenfunctions in bunches with space charge. Using numerical simulations, space-charge induced Landau damping in a bunch is demonstrated. The damping rates are quantified for different modes and space-charge tune shifts. Finally, the head-tail instability with space charge is studied for the resistive-wall impedance below the mode coupling threshold. Results demonstrate that space-charge induced damping can suppress the instability for moderately strong space charge; instability growth rates saturate at strong space charge, in agreement with theoretical predictions.
\end{abstract}

DOI: 10.1103/PhysRevSTAB.13.114201

PACS numbers: 29.20.D-

\section{INTRODUCTION}

Space charge shifts the frequency of the incoherent betatron oscillations which can change stability thresholds, growth rates, and real eigenfrequencies of transverse coherent instabilities. This is also true for the head-tail instability of ion bunches in ring machines. For the nominal beam parameters of high-intensity synchrotrons, as the projected SIS100 of FAIR [1], or the CERN PS, the bunch conditions correspond to strong space charge, $\Delta Q_{\mathrm{sc}} \gg Q_{s}$, or moderate space charge, $\Delta Q_{\mathrm{sc}} \gtrless Q_{s}$, where $\Delta Q_{\mathrm{sc}}$ is the peak shift of the betatron tune due to space charge and $Q_{s}$ is the synchrotron tune. The standard head-tail theory, i.e., the model of Sacherer [2,3], does not include the effect of an incoherent tune spread on head-tail modes.

A model for the head-tail instability with arbitrary space charge has been suggested in Ref. [4], for a bunch in a square-well potential and an airbag bunch distribution in the longitudinal phase space. Although being rather artificial at first glance, this model appears to be very useful to gain physical insight and for code validation. Only in recent works [5-7] analytical treatments of head-tail modes with space charge for realistic bunch distributions (as, e.g., Gaussian) have been proposed. However, numerical simulations appear to be indispensable for a comprehensive stability analysis in different beam parameter regimes and with various collective effects taken into account. Here, we present particle tracking simulations for head-tail modes in a Gaussian bunch with space charge. We use two different particle tracking codes, PATRIC [8] and HEADTAIL [9], in order to compare different numerical implementations. As an exemplary instability driving source, the resistive-wall impedance is considered. In this work we consider the single-bunch head-tail instability for the parameters well below the threshold for mode coupling.
An important phenomenon, discussed in Refs. [5-7], is Landau damping in a bunch exclusively due to space charge. In a coasting beam space charge cannot provide Landau damping of its own, even if the coherent frequency overlaps the tune spread induced by nonlinear space charge [10]. In the case of a bunch, the synchrotron motion plays an important role and the space-charge tune spread due to the longitudinal density profile provides Landau damping. Here, we demonstrate this Landau damping in particle tracking simulations and examine its role for the stability of head-tail modes at moderate and stronger space charge.

\section{BUNCH EIGENFREQUENCIES WITH SPACE CHARGE}

The eigenspectrum of transverse head-tail oscillations for a bunch in an rf bucket with the synchrotron tune $Q_{s}$ can be described by $Q_{k}=k Q_{s}(k \in \mathbb{Z})$, which can be observed in the Schottky noise as equidistant satellites at each betatron sideband. Collective interactions caused by ring impedances or space charge modify this spectrum and shift the eigenfrequencies of the head-tail modes. As a result the Landau damping strength can be modified; the growth rate of a head-tail instability can be changed, as well as the mode coupling thresholds. Space charge causes a decrease in the incoherent betatron tune depending on the particle position along the bunch, which can be described by the tune shift

$$
\Delta Q_{\mathrm{sc}}(\tau)=\frac{\lambda(\tau) r_{p} R^{2}}{\gamma^{3} \beta^{2} Q_{0} a^{2}},
$$

where $R$ is the ring radius, $\beta$ and $\gamma$ are relativistic parameters, $r_{p}=q_{\mathrm{ion}}^{2} / 4 \pi \epsilon_{0} m c^{2}$ is the classical particle radius, $a$ is the total beam radius, $\lambda$ is the beam line density, $\tau$ is the longitudinal distance in radian, and $Q_{0}$ is the bare betatron 


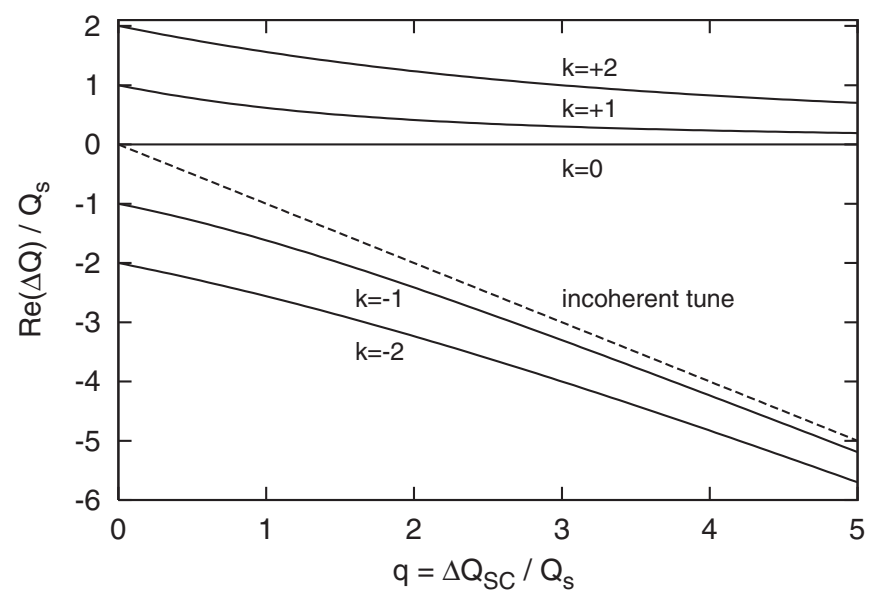

FIG. 1. Tune shifts of five head-tail modes versus space-charge parameter $q$ as given by the airbag theory [Eq. (2)]. The dashed line is the incoherent betatron tune $Q_{0}-\Delta Q_{\mathrm{sc}}$.

tune. This tune shift $\Delta Q_{\mathrm{sc}}$ is valid for a round cross section with the transverse K-V distribution and is defined as the modulus of the negative shift.

There is no simple analytical answer for the space charge effect on head-tail modes in bunches with an arbitrary bunch profile $\lambda(\tau)$. However, such a theory could be very useful for code validation and for the interpretation of simulation results. An analytical solution for head-tail modes in bunches with arbitrary space charge has been derived in Ref. [4]. The model assumes an airbag distribution in the longitudinal phase space and a square-well (or barrier) potential and thus a constant line density, which means a constant $\Delta Q_{\mathrm{sc}}$. The longitudinal momentum distribution has two opposing flows of particles $\left[\delta\left(v_{0}-v_{b}\right)+\delta\left(v_{0}+v_{b}\right)\right]$, the synchrotron tune in this bunch is $Q_{s}=v_{b} /\left(2 \tau_{b} R f_{0}\right)$, where $\tau_{b}$ is the full bunch length and $f_{0}$ is the revolution frequency. The model considers "rigid flows," i.e., only dipole oscillations without variation in the transverse distribution of the flows are included. It also assumes that all betatron tune shifts are small compared to the bare tune $|\Delta Q| \ll Q_{0}$. The resulting tune shift due to space charge (without impedances) is given by

$$
\Delta Q=-\frac{\Delta Q_{\mathrm{sc}}}{2} \pm \sqrt{\frac{\Delta Q_{\mathrm{sc}}^{2}}{4}+k^{2} Q_{\mathrm{s}}^{2}}
$$

where "+" is for modes $k \geq 0$. These space-charge tune shifts are illustrated in Fig. 1, where we introduce a spacecharge parameter $q=\Delta Q_{\mathrm{sc}} / Q_{s}$.

In order to verify the space-charge implementation for long-time simulations with a particle tracking code, we have introduced the barrier-airbag bunch distribution in both PATRIC and HEADTAIL codes. For the transverse space-charge force, the "frozen" electric field model was used, i.e., a fixed potential configuration which follows the mass center for each single slice. This approach is justified for the "rigid-slice" regime and can be considered as a reasonable approach for moderate and strong space charge $[5,11]$. A round transverse cross section and a homogeneous transverse beam profile (the $\mathrm{K}-\mathrm{V}$ distribution) were used in the simulations in this work. An excellent agreement between the airbag theory [Eq. (2)] and simulations has been achieved, a detailed description of the code validation was presented in Ref. [12].

For a realistic case, we consider a Gaussian bunch, i.e., a Gaussian line density profile and a Gaussian momentum distribution. Coherent oscillation spectra for bunches with $q=5$ and $q=20$ are shown in Fig. 2. The space-charge parameter $q$ is calculated for the peak value of $\Delta Q_{\mathrm{sc}}$ in the bunch center. Head-tail eigenfrequencies from the airbag theory are given in Fig. 2 with red dashed lines. The differences in the tune shifts between the Gaussian bunch and the airbag bunch are below $\approx 12 \%$ for $q=5$, and below $\approx 5 \%$ in the case of $q=20$. Especially for strong space charge the airbag theory [Eq. (2)] gives a surprisingly good prediction for the bunch eigenfrequencies, even in the case of a Gaussian bunch, which can also be seen using results of Refs. [5-7].
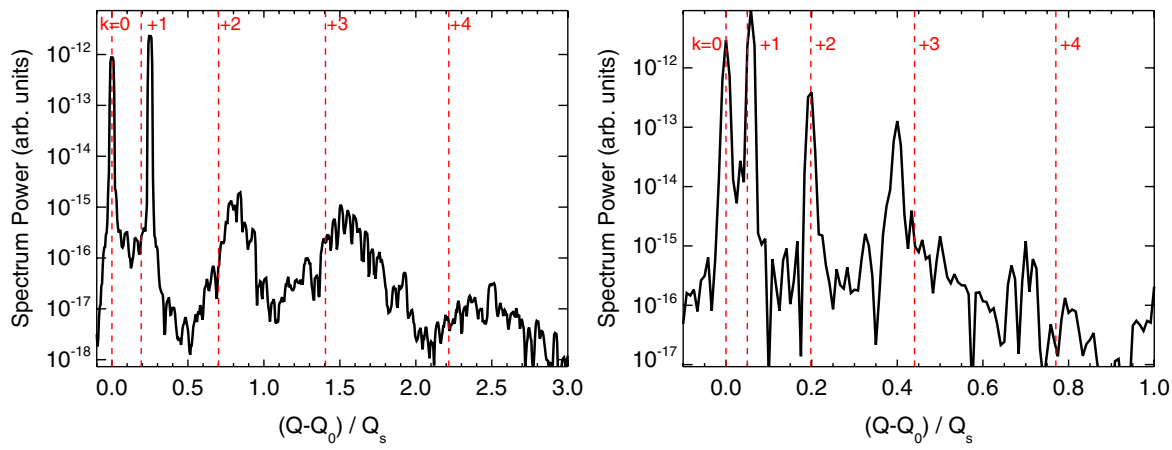

FIG. 2. Transverse bunch spectrum from simulations for a Gaussian bunch with space charge: left plot $q=5$, right plot $q=20$. Red dashed lines are head-tail modes for the airbag bunch, Eq. (2). To clarify the notation we note that the eigenfrequencies without space charge are $\left(Q-Q_{0}\right) / Q_{s}=1$ for $k=1,\left(Q-Q_{0}\right) / Q_{s}=2$ for $k=2$, etc. 


\section{LANDAU DAMPING DUE TO SPACE CHARGE}

In simulations for a Gaussian bunch we have observed Landau damping due to the effect of space charge. This kind of damping is provided by the variation of the spacecharge tune shift along the bunch which causes a tune spread. Note that this is opposite to a coasting beam, where space charge cannot produce Landau damping of its own. A regular exponential decrease of the mode amplitude in time has been observed. Results of a space-charge scan for the modes $k=1$ and $k=2$ are presented in Fig. 3 .

In order to characterize bunch Landau damping for different head-tail modes and bunch parameters, we consider an initial perturbation with an eigenmode. As a reasonable approximation, we trigger a $k$ mode of the airbag bunch [4] $\bar{x}_{k}(\tau)=A_{0} \exp (-i \zeta \tau) \cos \left(k \pi \tau / \tau_{b}\right)$ and follow the time evolution of the perturbation. Here $\zeta=$ $\xi Q_{0} / \eta$ is the normalized chromaticity, $\Delta Q_{\xi} / Q=$ $\xi \Delta p / p, \eta$ is the slip factor. Using our simulations it is possible to demonstrate that, on the one hand, the eigenmodes in a Gaussian bunch are very close to the airbag modes, and on the other hand, to compare these eigenmodes with the eigenfunctions obtained in Refs. [5,6]. For this, we start with the airbag $\bar{x}_{k}(\tau)$ for $k=1$ and $k=2$ with $q=6, \xi=0$ and observe the bunch dipole traces after approximately two damping times of $k=2$. A comparison of these numerical traces (red lines) with the airbag eigenmodes (blue lines) and with the eigenfunctions from $[5,6]$ (green lines) is presented in Fig. 4. For the airbag modes $\tau_{b}=4 \sigma_{z} / R$ was chosen, where $\sigma_{z}$ is the rms bunch length of the Gaussian bunch. Starting with the airbag $k=1$ mode, there is no contribution from $k=0$ and $k=2$ because it is an odd function. For $k=2$, the $k=1$ mode is excluded because it is an even function, and we exclude the $k=0$ mode by making the integrated bunch offset zero. Additionally we note that, in the case of a large difference between the true eigenmode and an approximation, this difference is given by modes of higher

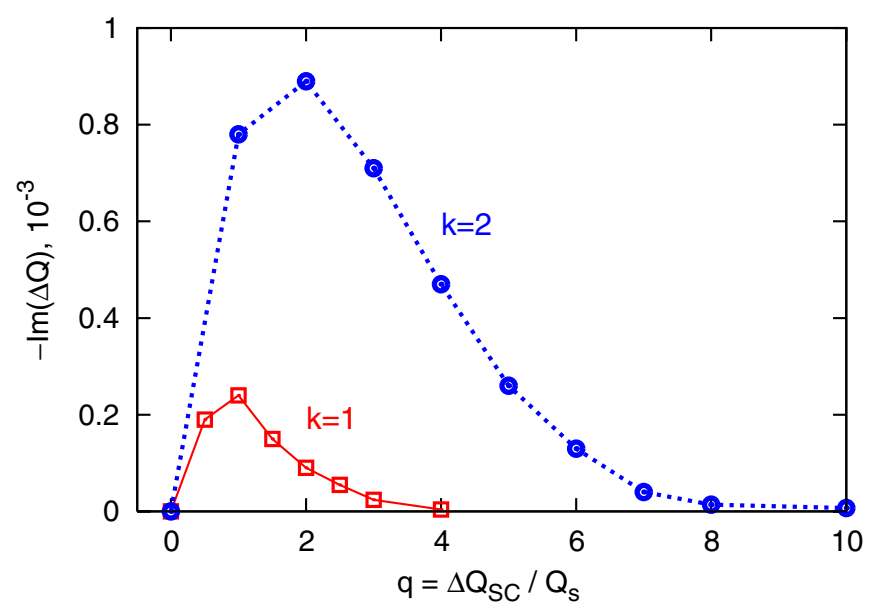

FIG. 3. Damping decrement of the $k=1$ and $k=2$ modes obtained from simulations for a Gaussian bunch, $Q_{s}=0.01$.

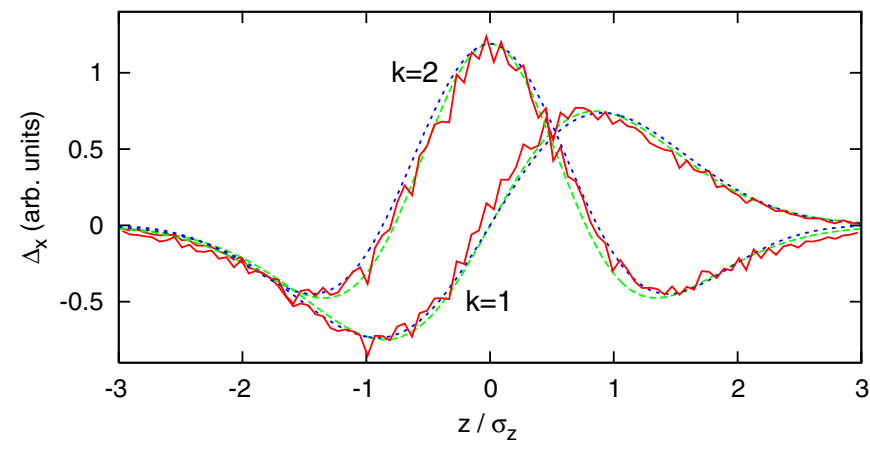

FIG. 4. Dipole moments from simulations for a Gaussian bunch (red lines), theory eigenfunctions for a Gaussian bunch from Refs. [5,6] (green lines), and dipole moments with the beam offset for analytical eigenmodes of an airbag bunch (blue lines).

$k$, which are Landau damped much faster than the mode considered.

Landau damping examples are shown in Fig. 5, where the momentum $M_{k}=\int \bar{x}_{\text {code }} \cos \left(k \pi \tau / \tau_{b}\right) d \tau$ is plotted turn by turn for two cases; here $\bar{x}_{\text {code }}(\tau)$ is the simulation output. Simulations for bunch truncations between $2 \sigma_{z}$ and $3 \sigma_{z}$ of the half length did not provide significant differences. For stronger space charge, the longitudinal bunch tails above $2 \sigma$ should be important for Landau damping.

It follows from the dimensionality that Landau damping in a bunch for a given $q$ scales linearly with the synchrotron tune. Figure 6 demonstrates results of a $Q_{s}$ scan for the modes $k=1$ and $k=2$, the linear lines in the plot cross the origin.

The effect of Landau damping can be also observed on the bunch spectra in Fig. 2. The modes $k=2$ and $k=3$ are strongly damped at $q=5$ (the left-hand side plot), which is not the case at $q=20$ (see the right-hand side plot in Fig. 2).

An estimation for the Landau damping rate $\operatorname{Im}(\Delta Q) \sim$ $-k^{4} Q_{s} / q^{3}$ has been obtained in Refs. $[5,6]$ for strong space charge, which was defined as $q \gg 2 k$. In this sense Landau

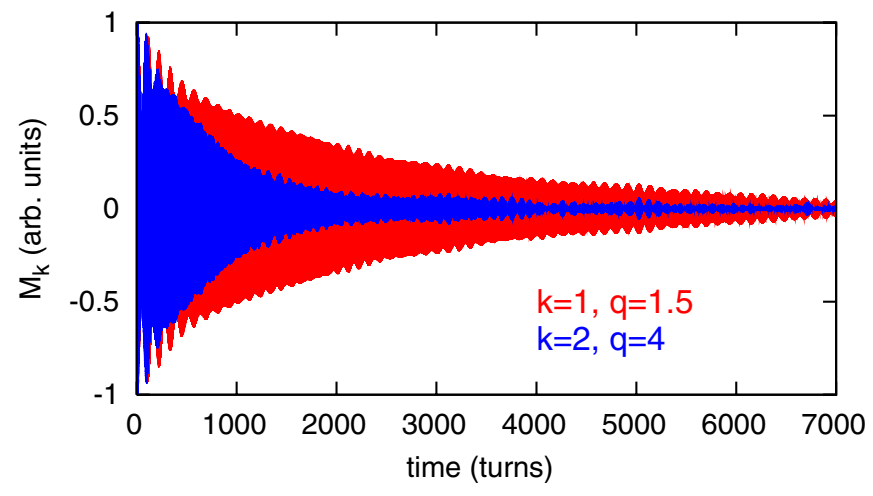

FIG. 5. Simulations with a Gaussian bunch: examples for Landau damping due to the space-charge effect in a bunch for $k=1$ (red line) and $k=2$ (blue line), $Q_{s}=0.004$. 


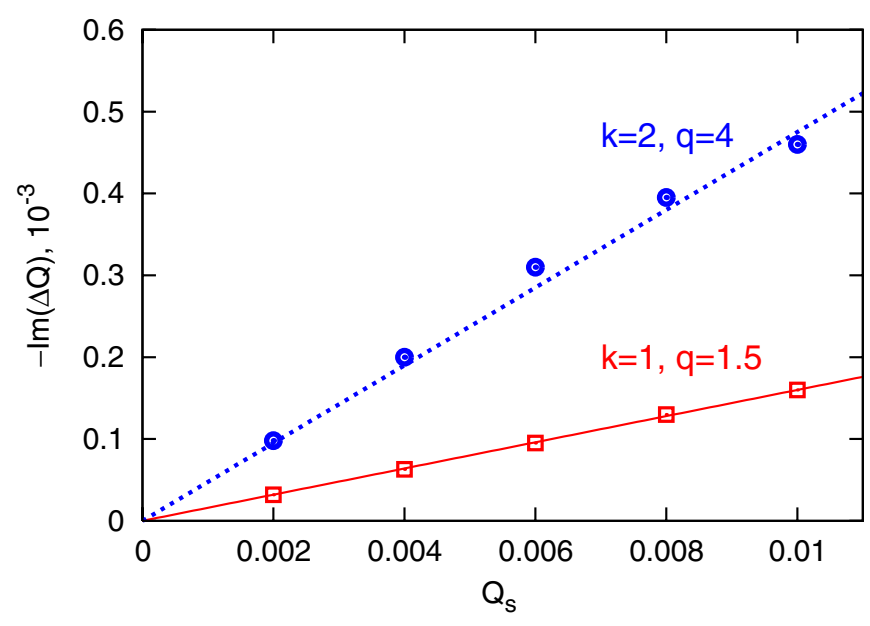

FIG. 6. Synchrotron tune dependence of the damping decrement for $k=1$ (squares) and $k=2$ (circles) modes obtained from simulations with a Gaussian bunch. The lines are linear and cross the origin.

damping demonstrated in our simulations relates to moderate space charge. Nevertheless, it is still interesting to compare some ultimate points of Fig. 3 with this estimation. This comparison shows a reasonable agreement for the dependence of the damping rate on the mode number and on the space-charge parameter. However, the absolute values for $\operatorname{Im}(\Delta Q)$ from our simulations are smaller by approximately an order of magnitude. Further simulations for stronger space charge should shed more light on this issue. Also the role of transverse nonlinear space charge in bunch Landau damping should be studied.

A physical interpretation for space-charge induced Landau damping is now discussed. The explanation is illustrated in Fig. 7. Because of the line density variation along the bunch, particles with different synchrotron amplitudes have different space-charge tune shifts. The Landau damping mechanism corresponds to the energy transfer between the coherent mode and the resonant particles. Considering the incoherent spectrum related to a chosen $k$ mode, it is easy to see that particles with large synchrotron amplitudes represent the resonant particles. These particles have small space-charge tune shifts, giving the upper boundary of the effective incoherent spectrum. This can be roughly estimated from the longitudinal average of the space-charge intensity. Assuming $\pm 2 \sigma_{z}$ as the relevant area for the efficient space-charge tune spread, and taking into account the modulation by the synchrotron motion [7],

$$
\Delta Q_{\max } \approx-0.23 Q_{s} q+k Q_{s},
$$

see Fig. 7. The lower boundary of the incoherent spectrum, which is located well below the coherent line, corresponds to the strongest space-charge tune shift and is represented by the particles with small synchrotron amplitudes. As we demonstrate in Fig. 2, the airbag theory is a good
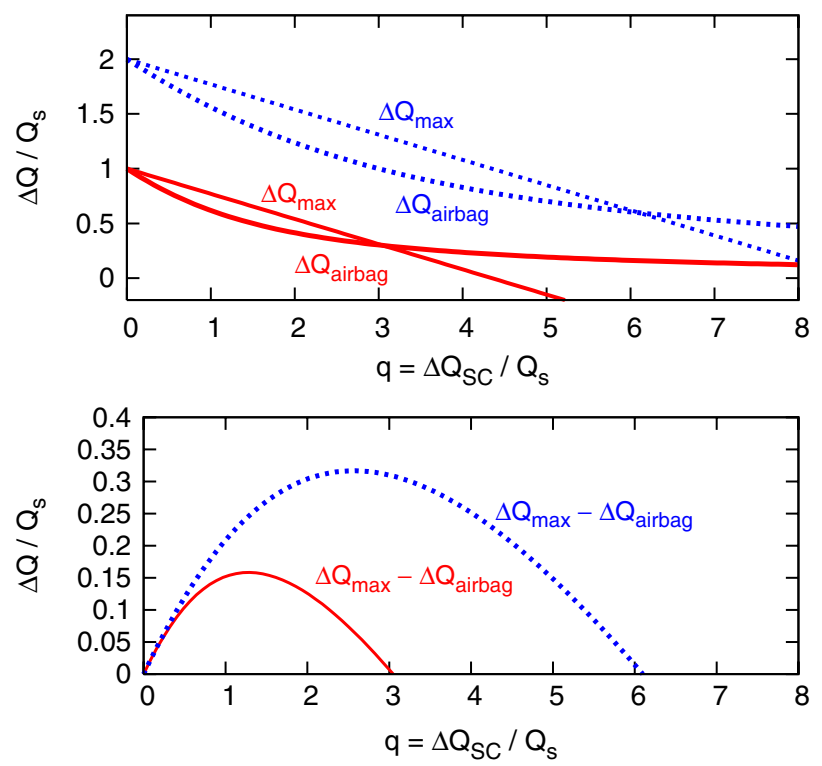

FIG. 7. Illustration for the active area of the bunch Landau damping with the betatron tune shifts as functions of the spacecharge parameter. The red lines correspond to the $k=1$ mode; the blue dashed lines show the $k=2$ mode. The parameter $\Delta Q_{\max }$ is the upper boundary of the effective spectrum and $\Delta Q_{\text {airbag }}$ is the eigenfrequency of the head-tail modes from the airbag theory [Eq. (2)].

approximation for the space-charge frequency shifts of head-tail eigenmodes even for a Gaussian bunch. If we suggest that transverse Landau damping due to space charge should be active when the coherent head-tail mode lies within the effective spectrum (or $\Delta Q_{\text {airbag }}$ is below $\Delta Q_{\max }$ in Fig. 7), we would expect the area of Landau damping to be as illustrated in the bottom plot of Fig. 7. Note that the dependencies of the damping rates on $q$ in Fig. 3 are qualitatively similar to the curves in the bottom plot of Fig. 7.

\section{HEAD-TAIL INSTABILITY WITH SPACE CHARGE}

The theory for head-tail modes in an airbag bunch [4] provides not only eigenfrequencies for modes with space charge, but also gives a simple analytical answer for the growth rates and for the real tune shifts of unstable headtail modes. This solution is obtained assuming the wake potential $W(\tau)=W_{0} \exp (-\alpha \tau)$ with a sufficiently short range compared to the bunch length, $\alpha \tau_{b} \gg 1$. For the mode $k=0$, which is not affected by space charge, the tune shift is given by

$$
\Delta Q=\Delta Q_{a b 0}(\alpha / \zeta+i)
$$

where we define $\Delta Q_{a b 0}$ as the growth rate for the mode $k=0$, 


$$
\Delta Q_{a b 0}=-\frac{\zeta}{\alpha^{2}} \kappa W_{0}
$$

and

$$
\kappa=\frac{\lambda_{0} q_{\mathrm{ion}}^{2}}{4 \pi \gamma m \omega_{0}^{2} Q_{0}} .
$$

For modes $k \neq 0$ this theory predicts

$$
\Delta Q=-\Delta Q_{\mathrm{sc}}+\frac{\Lambda_{0} \pm \sqrt{\Lambda_{0}^{2}+4 k^{2} Q_{s}^{2} \Lambda_{s}}}{2 \Lambda_{s}},
$$

where

$$
\begin{aligned}
& \Lambda_{0}=\Delta Q_{a b 0}(\alpha / \zeta+i)+\Delta Q_{\mathrm{sc}}, \\
& \Lambda_{\mathrm{s}}=1-\left[\frac{\Delta Q_{a b 0} \pi}{2 \zeta Q_{s} \tau_{b}}\right]^{2} .
\end{aligned}
$$

For the code verification in the case of head-tail modes with space charge, the exponential wake potential $W(\tau)=$ $W_{0} \exp (-\alpha \tau)$ has been implemented in the codes. The results of a parametric scan for the airbag bunch are presented in Fig. 8, where a constant space-charge tune shift $\Delta Q_{\mathrm{sc}}=3 Q_{s}$ was assumed, so the scan corresponds to an increasing impedance. In agreement with the theory our simulations demonstrate that space charge does not affect the $k=0$ mode, which is the most unstable head-tail mode. At the same time space charge strongly modifies $k \neq 0$ head-tail modes, which is seen in Fig. 8 for $k= \pm 1$
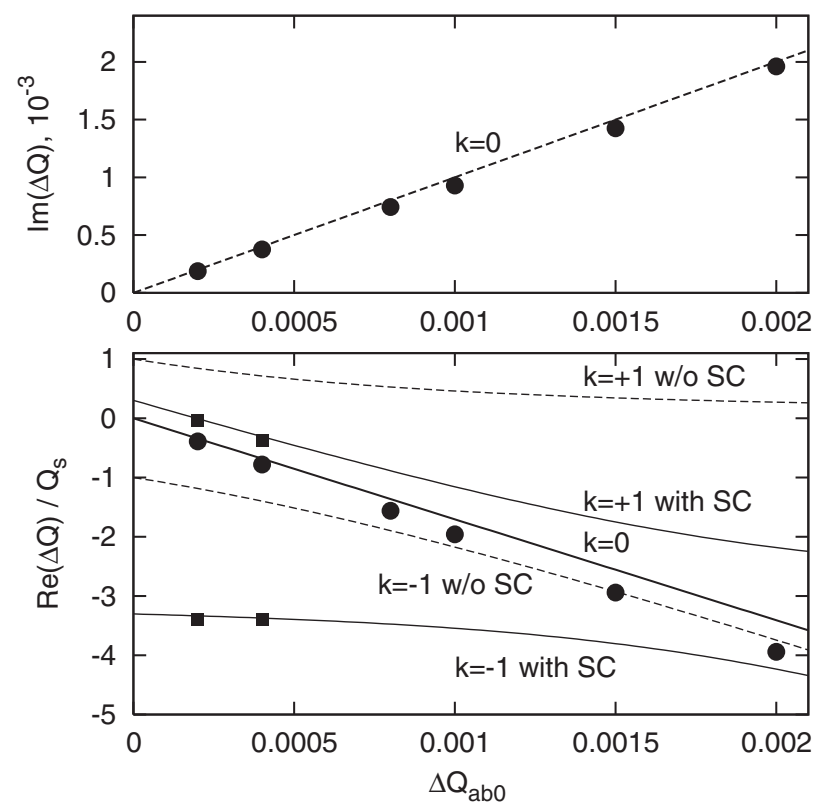

FIG. 8. Growth rates (upper plot) and real tune shifts (bottom plot) of head-tail modes in the airbag bunch with space charge $q=3$. Symbols: simulation output; lines: airbag theory [Eqs. (4) -(7)]. Tune shifts $\operatorname{Re}(\Delta Q)$ from simulations for $k= \pm 1$ (squares) are shown only for small $\Delta Q_{a b 0}$. The analytical results for cases with/without space charge are shown for comparison. modes for small wake amplitudes $W_{0}$, for which it was still possible to resolve real tune shifts of these modes. For high intensity, discrepancies appear due to the fact that the model breaks down. An increasing real impedance produces mode coupling which is not included in the analytic solution of the airbag model. Mode coupling also causes difficulties in the simulation of the head-tail instability with space charge. In the simulations, the impedance must be kept well below the threshold of mode coupling which requires long time scale (tens of thousands of turns) runs. The code validation has been presented in [12] in more detail.

We should point out an important property of head-tail modes which can be observed using the airbag bunch theory [4]. It concerns the effect of space charge, especially for strong space charge, on the growth rate and on the real frequency shift. Figure 9 shows three head-tail modes for increasing space charge, as given by the theory [Eqs. (4)(7)]. The growth rates are normalized by that of the $k=0$ mode. We observe a saturation of the space-charge effect, where the growth of $q$ does not change the mode tune shift after a certain level. The negative modes $k<0$, which are not shown in the plot, are increasingly damped with growing space charge, and $\operatorname{Im}(\Delta Q)$ of these modes goes to zero due to counterphase oscillations of the two flows [13].

The airbag bunch theory [4] is not appropriate for reliable estimations of the bunch stability mainly due to two reasons: the constant line density, which makes Landau damping impossible, and the short-range wake. The most unstable mode is thus always the $k=0$ mode for Eqs. (4)-(7). This is not true for the resistive-wall

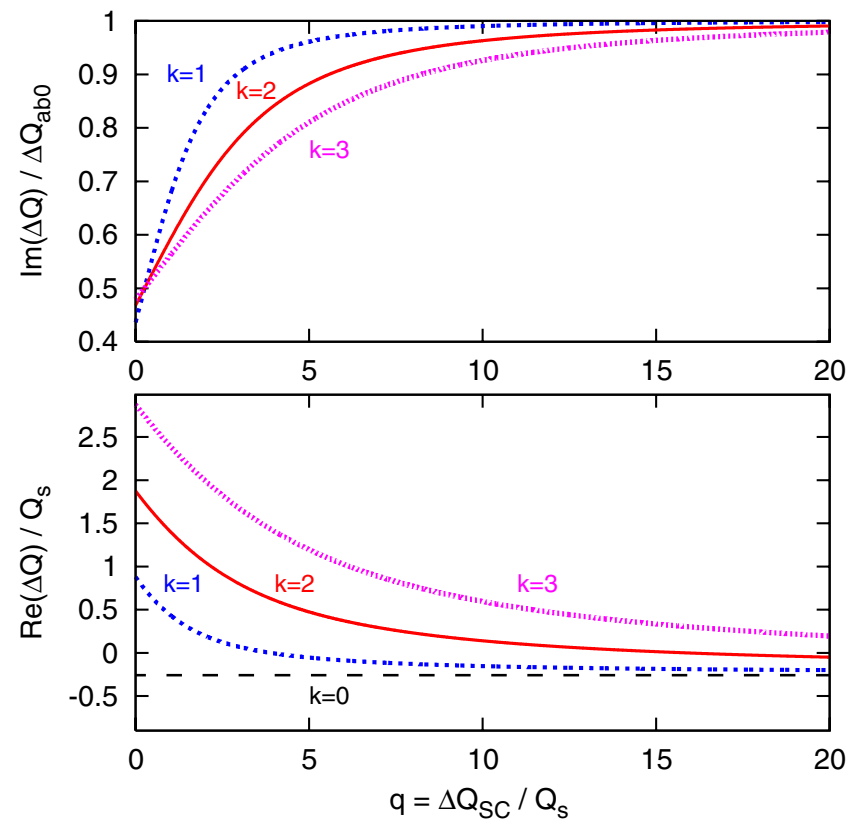

FIG. 9. Growth rates (upper plot) and real tune shifts (bottom plot) as given by the airbag theory [Eqs. (4)-(6)] in a dependence on the space-charge parameter. 
impedance, which gives a long-range wake and is usually one of the main coherent instability sources. According to the Sacherer theory [2], the mode index $k$ of the most unstable head-tail mode due to the resistive-wall impedance increases with the growing chromaticity modulus, which was also confirmed by measurements in the CERN PS synchrotron [14]. A similar result was obtained for bunches with strong space charge in $[5,6]$.

Hence, in our simulations we consider a more realistic situation with a Gaussian bunch and with the wake function of the thick resistive wall,

$$
W_{r w}(z)=-\frac{c L_{r w}}{b^{3}}\left(\frac{\beta}{\pi}\right)^{3 / 2} \sqrt{\frac{Z_{0}}{z \sigma_{r w}}},
$$

where $b$ is the pipe radius, $\sigma_{r w}$ is the conductivity, $L_{r w}$ is the pipe length, and $Z_{0}=376.7 \mathrm{Ohm}$; the effect of the wake is taken into account in the single-bunch regime, multiturn effects are not included. We consider a beam below transition, thus three exemplar negative chromaticities were considered. A synchrotron tune of $Q_{s}=0.01$ was chosen. Figure 10 summarizes results of our particle tracking simulations. Without space charge, at $q=0$, we obtain the head-tail modes $k=1, k=2$, and $k=3$ as the most unstable modes for the respective chromaticity, and examine the effect of increasing space charge for a constant impedance. A simulation is started with a nondisturbed bunch and the instability development is observed, thus this method provides only positive $\operatorname{Im}(\Delta Q)$, the simulation points inside of the stable areas are not shown for simplicity. The first important observation is that for moderate space-charge Landau damping suppresses all the head-tail modes, in agreement with the results of the previous section. Second, for large $\Delta Q_{\mathrm{sc}}$, above the tune shift range for strong Landau damping, the growth rates do

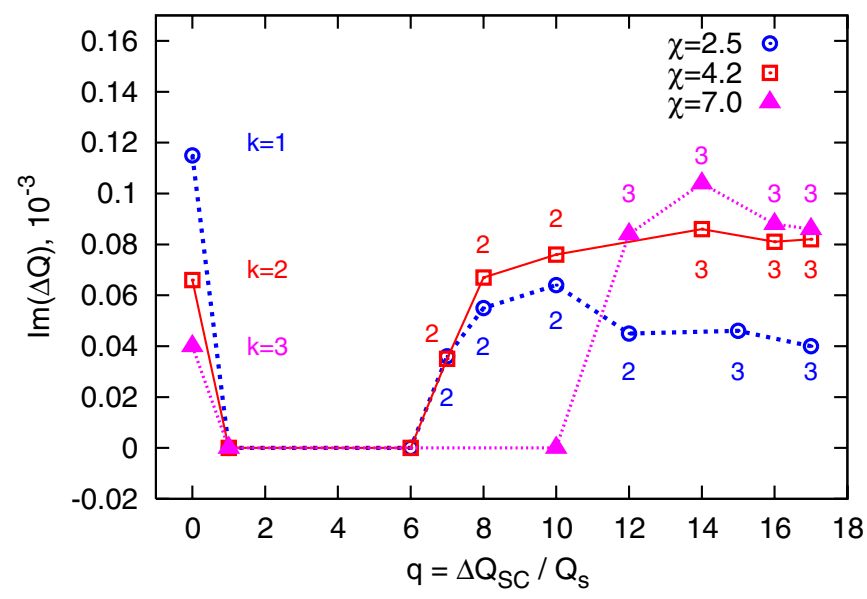

FIG. 10. Growth rates of the most unstable head-tail modes obtained in simulations for a Gaussian bunch for three different head-tail phases $\chi=\zeta \tau_{b}$ in a dependence on the space-charge parameter. The mode index $k$ is given for each data point with the corresponding color. not experience significant changes with increasing space charge. At the same time, lowest-order modes $(k=1$ and $k=2$ ) leave the role of the strongest head-tail instability to higher-order modes. An example of the most prominent modes for different space-charge strengths is shown in Fig. 11.

The observation of the growth rate saturation for strong space charge has already been made in the analytic airbag theory for the short-range exponential wake, see Fig. 9. However, Landau damping does not appear in this case due to a constant line density.

On the other hand, the behavior of head-tail modes at strong space charge can be understood in terms of the calculations in Refs. [5,6], where the author argues that treating a wake as a perturbation provides the related tune shift in the form of a diagonal element of the wake operator,

$$
\Delta Q=\frac{\kappa}{N_{\text {ion }} \lambda_{0} R} \int_{0}^{z_{b}} d z \int_{z}^{z_{b}} d s W(s-z) d_{k}(s) d_{k}^{*}(z),
$$

where $d_{k}(s)=\lambda(s) \bar{x}_{k}(s), \int \lambda(s) d s=N_{\text {ion. }}$. In the case of the airbag bunch, where the eigenfunctions do not depend on space charge, this means that space charge has no effect on $\Delta Q$. Figure 9 confirms this statement for strong space charge. Also for an arbitrary bunch profile, e.g., Gaussian, the space-charge induced deformation of the eigenfunctions is small at strong space charge [5-7]. Hence, the tune shift should saturate with increasing space charge and Eq. (9) should give an estimation for the mode growth
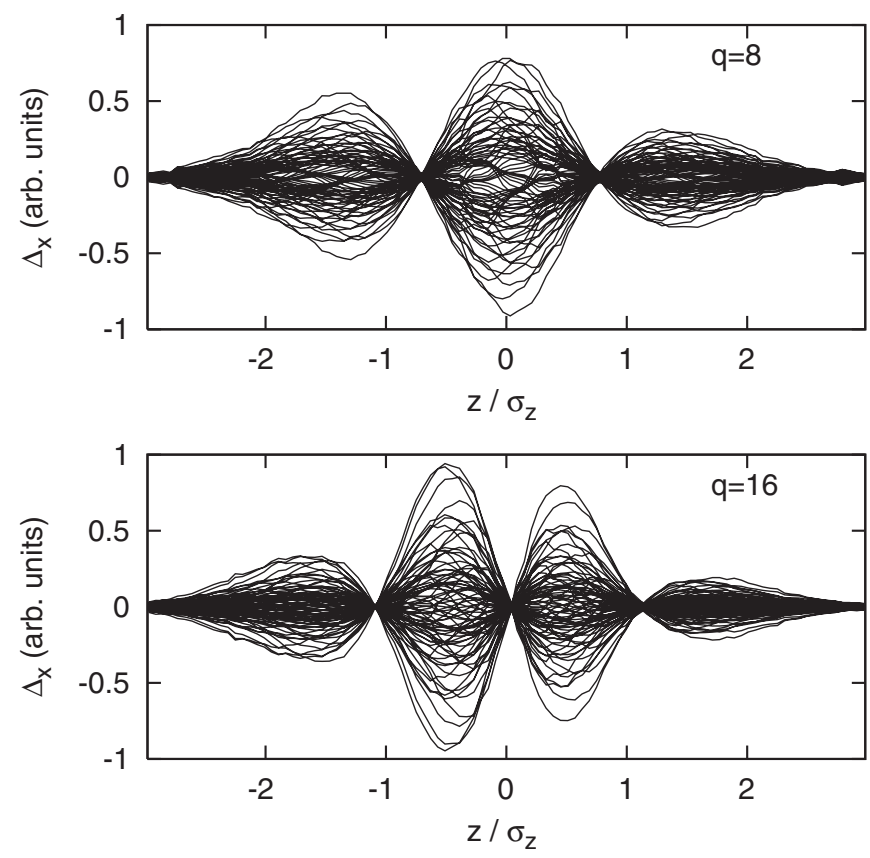

FIG. 11. Examples of bunch dipole traces of head-tail instabilities from Fig. 10 for the head-tail phase $\chi=4$.2. The upper plot for $q=8$ demonstrates the $k=2$ mode, while the lower plot for $q=16$ shows the $k=3$ mode. 
rate at saturation. Using this expression for the wake [Eq. (8)] and for beam parameters in our simulations, we obtain for the most unstable modes $\operatorname{Im}(\Delta Q)=$ $0.06 \times 10^{-3}(k=2)$ for $\chi=4.2$ and $\operatorname{Im}(\Delta Q)=0.055 \times$ $10^{-3}(k=3)$ for $\chi=7.0$. Hence, we find a reasonable agreement between our simulations and the ansatz Eq. (9) for estimations of the head-tail instability growth rates at strong space charge.

\section{CONCLUSIONS}

The effect of space charge on the weak head-tail instability has been studied using particle tracking simulations. An analytical theory [4] for an airbag bunch with a shortrange wake was employed for code validation and for the interpretation of realistic simulation results. The airbag theory gives a good prediction of the bunch eigenfrequencies, even in the case of a realistic Gaussian bunch. It has been demonstrated that the transverse eigenfunctions in a Gaussian bunch with space charge correspond to eigenmodes obtained in Refs. [5,6], which, interestingly, are also very close to the airbag [4] eigenmodes.

Landau damping of head-tail modes exclusively due to transverse space charge was demonstrated in simulations for a Gaussian bunch. The range of the space-charge strength where Landau damping is mostly prominent depends on the mode index $k$ and can be understood using a simple argumentation in terms of the mode eigenfrequency and the band of incoherent frequencies.

Simulations of the head-tail instability with space charge and the resistive-wall impedance showed that Landau damping can effectively stabilize the bunch at moderate space charge. In agreement with the airbag theory and with the results of Refs. [5,6], the instability growth rates saturate at strong space charge. Absolute values of growth rates are in a good agreement with the method $[5,6]$ to estimate the head-tail instability growth rates at strong space charge using a diagonal element of the wake operator [Eq. (9)].

Applying our results to experimental observations in existing machines and to future machines we discuss two interesting examples. The head-tail instabilities observed in CERN PS correspond rather well to the Sacherer theory [2], as reported in [14]. Indeed, the space-charge parameter for bunches in CERN PS is of order of $q \approx 150$, which is far above the range of Landau damping for observed modes. On the other hand, at such a strong space charge the growth rates of head-tail modes should be saturated and should not be very different from the no-space-charge estimation. Another example concerns the nominal parameters for uranium bunches in SIS100 [1,15]. Here the space-charge parameter lies in the range of $q \approx 20$. Landau damping might then give a significant contribution to the stability of the head-tail modes.

\section{ACKNOWLEDGMENTS}

We are grateful to Alexey Burov (FNAL) for useful discussions and for his comments to the paper script. We thank Giovanni Rumolo (CERN) for fruitful collaboration.

[1] FAIR Baseline Technical Report 2006, edited by H. H. Gutbrod.

[2] F. Sacherer, in Proceedings of the First International School of Particle Accelerators, Erice (1976), p. 198.

[3] F. Sacherer, CERN Report No. CERN/SI-BR/72-5, 1972.

[4] M. Blaskiewicz, Phys. Rev. ST Accel. Beams 1, 044201 (1998).

[5] A. Burov, Phys. Rev. ST Accel. Beams 12, 044202 (2009).

[6] A. Burov, Phys. Rev. ST Accel. Beams 12, 109901(E) (2009).

[7] V. Balbekov, Phys. Rev. ST Accel. Beams 12, 124402 (2009).

[8] O. Boine-Frankenheim and V. Kornilov, in Proceedings of the ICAP2006, Chamonix Mont-Blanc (2006).

[9] G. Rumolo and F. Zimmermann, Phys. Rev. ST Accel. Beams 5, 121002 (2002)

[10] V. Kornilov, O. Boine-Frankenheim, and I. Hofmann, Phys. Rev. ST Accel. Beams 11, 014201 (2008).

[11] A. Burov and V. Lebedev, Phys. Rev. ST Accel. Beams 12, 034201 (2009).

[12] V. Kornilov and O. Boine-Frankenheim, in Proceedings of ICAP2009, San Francisco (2009).

[13] O. Boine-Frankenheim and V. Kornilov, Phys. Rev. ST Accel. Beams 12, 114201 (2009).

[14] E. Métral, G. Rumolo, R. Steerenberg, and B. Salvant, in Proceedings of the 2007 Particle Accelerator Conference, Albuquerque, New Mexico (IEEE, Albuquerque, New Mexico, 2007), p. 4210.

[15] V. Kornilov, O. Boine-Frankenheim, and I. Hofmann, GSI Technical Report No. GSI-Acc-Note-2008-006, GSI Darmstadt, 2008. 